\title{
Local-channel-induced rise of quantum correlations in continuous-variable systems
}

\author{
Francesco Ciccarello and Vittorio Giovannetti \\ NEST, Scuola Normale Superiore, and Istituto Nanoscienze-CNR, Piazza dei Cavalieri 7, I-56126 Pisa, Italy
}

(Received 7 October 2011; published 7 February 2012)

\begin{abstract}
It was recently discovered that the quantum correlations of a pair of disentangled qubits, as measured by the quantum discord, can increase solely because of their interaction with a local dissipative bath. Here, we show that a similar phenomenon can occur in continuous-variable bipartite systems. With this aim, we consider a class of two-mode squeezed thermal states and study the behavior of Gaussian quantum discord under various local Markovian nonunitary channels. While these in general cause a monotonic drop of quantum correlations, an initial rise can take place with a thermal-noise channel.
\end{abstract}

DOI: 10.1103/PhysRevA.85.022108

PACS number(s): 03.65.Ud, 03.67.Mn, 03.65.Yz

\section{INTRODUCTION}

Within the remit of quantum-information processing (QIP) theory [1] and beyond, a paramount topic is the study of quantum correlations (QCs). Until recently, the emergence of QCs has been regularly highlighted in connection with nonseparable states of multipartite quantum systems [2]. The violation of the celebrated Bell inequalities is a typical signature of the extra amount of correlations that quantum systems can possess in addition to those of a purely classical nature $[1,2]$. Nonseparability, i.e., entanglement, which relies on the superposition principle, has been in fact regarded as a necessary prerequisite in order for QCs to occur. In 2001, however, a new way was discovered [3] in which the superposition principle can entail an alternative type of nonclassical correlation even in the absence of any entanglement. Typical instances are mixtures of pure separable states which are locally nonorthogonal, i.e., indistinguishable [4]. More rigorously, a state has nonclassical correlations whenever its associated density operator cannot be diagonalized in a basis which is the tensor product of local orthonormal bases [5]. When this occurs the entire content of the correlations cannot be retrieved by any local measurement, at variance with the classical framework where this is always achievable. Following this breakthrough, a growing interest has developed, especially after the realization that this new QC paradigm could be key to some known entanglement-free QIP schemes [6].

Various measures have been proposed in the literature to detect such QCs. A prominent one is quantum discord [3], whose definition simply arises from the quantum generalization of two classically equivalent versions of the mutual information (one being based on the conditional entropy). These are found to differ in the quantum framework, the corresponding discrepancy just being the quantum discord. This encompasses both QCs associated with entanglement and those that can be exhibited by separable states. The nonequivalence between two such forms of nonclassical correlation arises when mixed states are addressed since as long as the state is pure the discord coincides with the entropy of entanglement.

Significant motivations to pursue a deeper understanding of these problems are currently being provided by various investigations targeting nonunitary dynamics. While entanglement is known to be extremely fragile to environmental interactions, QCs-as given by this extended notion - are generally very robust and in some cases even fully insensitive to phase noise [7]. This is related to the fact that zero-discord states are a set of negligible measure within the entire Hilbert space [8]. Still in the framework of nonunitary dynamics, very recently it was found that the interaction with a local and memoryless bath can even create QCs initially fully absent [9-12]. Such an effect, which is unattainable with entanglement [2], was shown to take place in particular for qubits prepared in a fully classical state and undergoing a local amplitude-damping channel. This describes the dissipative interaction with a local bath (such as the spontaneous emission of a two-level atom) [1]. Specifically, the behavior consists of an initial rise of QCs until a maximum is reached, followed by a slow decay, the entanglement being zero throughout. The essential underlying mechanism is that such a nonunitary process can map orthogonal states of a subsystem onto nonorthogonal ones. The state thus can no longer be diagonalized in a tensor product of orthonormal bases, i.e., it acquires QCs $[9,11]$.

Most of the work carried out so far along these lines, though, tackled qubits. It is however natural to wonder how the above findings are generalized for quantum continuous-variable (CV) systems, which routinely occur in quantum optics. Such investigations are still in their infancy. Indeed, the first measure of generalized QCs for Gaussian states of bipartite CV systems was worked out only in 2010 [13,14]. This quantity, called the Gaussian discord, is basically defined in the same spirit as standard discord [3] and likewise can be nonzero for separable states $[13,14]$. Recently, another measure has been proposed [15]. To date, only a few studies [13,16,17] have targeted the nonunitary dynamics of Gaussian discord in the presence of environmental interactions, including one model of a non-Markovian reservoir [18]. It was found that apart from the case of a common reservoir the nonunitary dynamics is detrimental to QCs $[13,16,17]$.

Our goal in this work is to analyze the behavior of Gaussian discord in the case of two CV modes under the most relevant known instances of local-i.e., single-modeGaussian memoryless channels [19-21] each described by an associated completely positive quantum map. Our major motivation is to assess whether growth of QCs can take place for some local nonunitary channels as in the case of qubits [9-11]. We will indeed find that, in significant analogy with the qubits' framework, this can occur with a lossy channel (more generally with low-temperature thermal-noise channels). 
This paper is organized as follows. In Sec. II, we briefly review the definition of Gaussian discord. In Sec. III, we describe the class of squeezed thermal states, which is the one that will be our focus in this work. In Sec. IV, we review each of the aforementioned Gaussian channels and the related main features to which we will refer. In Sec. V, we show the behavior of QCs under each of the considered channels for some paradigmatic initial states. In Sec. VI, we provide a simple picture that allows us to obtain insight into the effects presented in the previous section. Finally, in Sec. VII we draw our conclusions.

\section{QUANTUM DISCORD OF GAUSSIAN STATES: REVIEW}

Given two systems 1 and 2 in a state $\rho$, the quantum mutual information is defined as

$$
\mathcal{I}(\rho)=S\left(\rho_{1}\right)+S\left(\rho_{2}\right)-S(\rho),
$$

where $\rho_{1(2)}=\operatorname{Tr}_{2(1)} \rho$ is the reduced density operator describing the state of 1 (2) and $S(\sigma)=-\operatorname{Tr}\left(\sigma \log _{2} \sigma\right)$ is the von Neumann entropy of an arbitrary state $\sigma$. A local generalized measurement on 2 can be specified by a complete set of positive-operator-valued projectors or measures (POVMs) $\left\{\Pi_{k}\right\}$, where $k$ indexes a possible outcome. If $k$ is recorded with probability $p_{k}=\operatorname{Tr}\left[\rho \mathbb{1} \otimes \Pi_{k}\right]$ the overall system collapses onto the (normalized) state $\rho_{k}=\left(\rho \mathbb{1} \otimes \Pi_{k}\right) / p_{k}$. The maximum of $S\left(\rho_{1}\right)-\sum_{k} p_{k} S\left(\rho_{k}\right)$, i.e., the mismatch between the entropy of 1 and the average conditional entropy, reads

$$
\mathcal{J}(\rho)=\max _{\left\{\Pi_{k}\right\}}\left[S\left(\rho_{1}\right)-\sum_{k} p_{k} S\left(\rho_{k}\right)\right],
$$

and is taken over all possible POVM measurements each described by the operator $\Pi_{k}$. If 1 and 2 are $C V$ modes and one restricts consideration to generalized Gaussian measurements [22] the Gaussian discord $\mathcal{D}^{\leftarrow}$ is defined as the discrepancy between (1) and (2) [13,14]:

$$
\mathcal{D}^{\leftarrow}=\mathcal{I}-\mathcal{J} .
$$

The arrow reminds us that measurements over mode 2 have been considered.

As is well known, any two-mode zero-mean Gaussian state $\rho_{12}$ is fully specified by its covariance matrix $\sigma_{i j}=$ $\operatorname{Tr}\left[\rho_{12}\left(R_{i} R_{j}+R_{j} R_{i}\right)\right]$, where $\boldsymbol{R}=\left\{x_{1}, p_{1}, x_{2}, p_{2}\right\}$ is the set of operators corresponding to the phase-space coordinates. Up to local symplectic, i.e., unitary, operations, the covariance matrix can be arranged in the form

$$
\sigma=\left(\begin{array}{ll}
A & C \\
C & B
\end{array}\right),
$$

where $A=\operatorname{diag}(a, a), B=\operatorname{diag}(b, b)$, and $C=\operatorname{diag}\left(c_{1}, c_{2}\right)$. The determinants of such diagonal matrices, $I_{1}=\operatorname{det} A$, $I_{2}=\operatorname{det} B, I_{3}=\operatorname{det} C$ along with $I_{4}=\operatorname{det} \sigma$ are symplectic invariants, i.e., they are invariant under local symplectic transformations. The quantum discord $\mathcal{D}^{\leftarrow}$ is a function of these symplectic invariants according to $[13,14]$

$$
\begin{aligned}
\mathcal{D}^{\leftarrow}= & h\left(\sqrt{I_{2}}\right)-h\left(d_{-}\right)-h\left(d_{+}\right) \\
& +h\left(\frac{\sqrt{I_{1}}+2 \sqrt{I_{1} I_{2}}+2 I_{3}}{1+2 \sqrt{I_{2}}}\right),
\end{aligned}
$$

where $h(x)=(x+1 / 2) \ln (x+1 / 2)-(x-1 / 2) \ln (x-1 / 2)$ and

$$
d_{ \pm}^{2}=\frac{\Delta \pm \sqrt{\Delta^{2}-4 I_{4}}}{2}
$$

with $\Delta=I_{1}+I_{2}+2 I_{3}$. The discord in terms of measurements on mode $1, \mathcal{D}^{\rightarrow}$, is obtained from (5) upon the replacement $I_{1} \leftrightarrow I_{2}$. In the remainder of this work, we will be solely interested in the discord (5); hence we will drop the subscript henceforth and set $\mathcal{D}=\mathcal{D}^{\leftarrow}$.

\section{TWO-MODE SQUEEZED THERMAL STATES}

In this work, we shall focus on the class of two-mode squeezed thermal states (STSs) whose generic element reads

$$
\rho=e^{r\left(a_{1}^{\dagger} a_{2}^{\dagger}-a_{1} a_{2}\right)}\left(\rho_{1} \otimes \rho_{2}\right)\left[e^{r\left(a_{1}^{\dagger} a_{2}^{\dagger}-a_{1} a_{2}\right)}\right]^{\dagger},
$$

where each single-mode thermal state $\rho_{i}(i=1,2)$ is given by

$$
\rho_{i}=\sum_{n=0}^{\infty} \frac{N_{i}^{n}}{\left(1+N_{i}\right)^{n+1}}|n\rangle_{i}\langle n|,
$$

with $N_{i}$ the corresponding average number of photons.

The characteristic function $\chi\left(\lambda_{1}, \lambda_{2}\right)$ [23] is obtained from (7) as

$$
\chi\left(\lambda_{1}, \lambda_{2}\right)=\operatorname{Tr}\left[\rho D\left(\lambda_{1}\right) D\left(\lambda_{2}\right)\right],
$$

where $D\left(\lambda_{i}\right)=\exp \left(\lambda_{i} a_{i}^{\dagger}-\lambda^{*} a_{i}\right)$ is the displacement operator of the $i$ th mode ( $i=1,2$ with $a_{i}$ and $a_{i}^{\dagger}$ the usual ladder operators of the $i$ th mode). The density operator is retrieved from the characteristic function as $\rho=$ $\int d^{2} \lambda_{1} d^{2} \lambda_{2} \chi\left(\lambda_{1}, \lambda_{2}\right) D\left(-\lambda_{1}\right) D\left(-\lambda_{2}\right) / \pi^{2}$. It is then straightforwardly checked that

$$
\begin{aligned}
\chi\left(\lambda_{1}, \lambda_{2}\right)= & e^{-\left(N_{1}+1 / 2\right)\left|\cosh r \lambda_{1}-\sinh r \lambda_{2}^{*}\right|^{2}} \\
& \times e^{-\left(N_{2}+1 / 2\right)\left|\cosh r \lambda_{2}-\sinh r \lambda_{1}^{*}\right|^{2}} .
\end{aligned}
$$

As states (7) are Gaussian, the covariance matrix is related to the characteristic function according to [24]

$$
\chi(\Lambda)=\exp \left[-\frac{\Lambda^{\mathrm{T}} \boldsymbol{\sigma} \Lambda}{2}\right],
$$

where $\Lambda=\left(\alpha_{1}, \beta_{1}, \alpha_{2}, \beta_{2}\right)^{\mathrm{T}}$ and we have decomposed each complex variable $\lambda_{j}(j=1,2)$ as $\lambda_{j}=\left(\alpha_{j}+i \beta_{j}\right) / \sqrt{2}$. Upon comparison between Eqs. (10) and (11) and in the light of (4), the diagonal matrix elements defining $A, B$, and $C$ [cf. Eq. (4)] are obtained as

$$
\begin{gathered}
a=\left(1+N_{r}\right) N_{1}+N_{r} N_{2}+N_{r}+1 / 2, \\
b=N_{r} N_{1}+\left(1+N_{r}\right) N_{2}+N_{r}+1 / 2, \\
c_{1}=-c_{2}=-\left(1+N_{1}+N_{2}\right) \sqrt{N_{r}\left(1+N_{r}\right),}
\end{gathered}
$$

where we have set $N_{r}=(\sinh r)^{2}$.

\section{LOCAL GAUSSIAN CHANNELS}

In this section, we review the salient features of the local one-mode Gaussian channels whose effect on the quantum correlations of two-mode states we aim to scrutinize. Throughout, we will assume that each of such channels acts 
on mode 2 only. The channels are Gaussian in that each of these maps Gaussian states into Gaussian states, hence allowing for use of Gaussian discord $[13,14]$ to shed light onto the QCs' behavior. Specifically, we address the thermal-noise channel, which reduces to the lossy channel in the limit of vanishing reservoir temperature, the amplifier channel, and the classical-noise channel [20].

\section{A. Thermal-noise channel}

This channel describes the dissipative interaction of a single-mode CV system with an environment at thermal equilibrium. Indeed, its associated map is routinely worked out by assuming that a single-mode environment in a thermal state specified by the average photon number $N$ is mixed by a beam splitter with the single-mode system. The state of the latter, and hence the map describing the channel, can then be obtained by simply tracing out the environmental degree of freedom. The channel quantum efficiency is measured by the parameter $\eta$ such that $0 \leqslant \eta \leqslant 1$ (in the above model $\eta$ is the transmissivity of the beam splitter).

Under the thermal-noise channel of efficiency $\eta$ and the environment's average photon number $N$, the characteristic function $\chi\left(\lambda_{1}, \lambda_{2}\right)$ transforms into $\chi^{\prime}\left(\lambda_{1}, \lambda_{2}\right)$ according to [25]

$$
x \chi^{\prime}\left(\lambda_{1}, \lambda_{2}\right)=\chi\left(\lambda_{1}, \sqrt{\eta} \lambda_{2}\right) e^{-(1-\eta)(N+1 / 2)\left|\lambda_{2}\right|^{2}} \quad(0 \leqslant \eta \leqslant 1) .
$$

For $N=0$ (zero-temperature environment) Eq. (15) reduces to the case of a lossy channel [20] acting on mode 2 .

By substituting (10) in Eq. (11) and comparing this with Eq. (15), it is straightforwardly found that the covariance matrix keeps the same structure as in Eq. (4) with $A=$ $\operatorname{diag}\left(a^{\prime}, a^{\prime}\right), B=\operatorname{diag}\left(b^{\prime}, b^{\prime}\right)$, and $C=\operatorname{diag}\left(c_{1}^{\prime}, c_{2}^{\prime}\right)$. The new diagonal matrix elements $\left\{a^{\prime}, b^{\prime}, c^{\prime}\right\}$ are related to the input ones [cf. Eqs. (12)-(14)] according to

$$
\begin{gathered}
a^{\prime}=a, \\
b^{\prime}=\eta b+(1-\eta)\left(N+\frac{1}{2}\right), \\
c_{1}^{\prime}=-c_{2}^{\prime}=\sqrt{\eta} c_{1} .
\end{gathered}
$$

\section{B. Amplifier channel}

This channel shares features similar to those of the thermal-noise channel but with the essential difference that it brings about an intensity amplification instead of a damping. Its corresponding map changes the characteristic function according to [26]

$$
\chi^{\prime}\left(\lambda_{1}, \lambda_{2}\right)=\chi\left(\lambda_{1}, \sqrt{k} \lambda_{2}\right) e^{-(k-1)(N+1 / 2)\left|\lambda_{2}\right|^{2}} \quad(k \geqslant 1),
$$

where $k \geqslant 1$ is a gain parameter. In the limit $k=1$ the channel reduces to the identity operator. By proceeding analogously to the case of the thermal-noise channel, we find that the covariance matrix has again the same form as in Eq. (4) with the diagonal matrix elements now given by

$$
\begin{gathered}
a^{\prime}=a, \\
b^{\prime}=k b+(k-1)\left(N+\frac{1}{2}\right), \\
c_{1}^{\prime}=-c_{2}^{\prime}=\sqrt{k} c_{1} .
\end{gathered}
$$

\section{Classical-noise channel}

This channel arises when classical Gaussian noise is superimposed on the single-mode system [27]. The parameter on which it depends is the number of injected noise photons $n \geqslant 0$ in such a way that the corresponding map becomes the identity in the limit $n=0$. The characteristic function is transformed under this channel according to

$$
\chi^{\prime}\left(\lambda_{1}, \lambda_{2}\right)=\chi\left(\lambda_{1}, \lambda_{2}\right) e^{-n\left|\lambda_{2}\right|^{2}} \quad(n \geqslant 0) .
$$

By comparing this with Eqs. (10) and (11) it turns out that the covariance matrix has the form (4) with diagonal matrix elements

$$
\begin{gathered}
a^{\prime}=a, \\
b^{\prime}=b+n, \\
c_{1}^{\prime}=-c_{2}^{\prime}=c_{1} .
\end{gathered}
$$

\section{BEHAVIOR OF QUANTUM CORRELATIONS}

Our aim in this section is to present some typical behaviors of Gaussian discord when states of the family (7) are subject to the local Gaussian channels introduced in the previous section. Unfortunately, a general analysis is quite demanding owing to the complicated functional form of (5). Nonetheless, as will become clear, one can obtain significant insight by addressing some paradigmatic instances. To begin with, we consider initial STSs [cf. Eqs. (7) and (8)] having $r=1$ and $N_{1}=N_{2}$. In Fig. 1, we plot the behavior of Gaussian discord (5) for increasing values of $N_{1}=N_{2}$ in the presence of the thermal-noise and amplifier channels (both with $N=0$ ) as well as the classical-noise channel. We recall that each local nonunitary map affects only mode 2 . The classical-noise channel [see Fig. 1(c)] has a merely detrimental effect on the QCs, which monotonically decay for a growing number of noise photons regardless of $N_{1}$. A similar effect is exhibited in the case of the amplifier channel when the gain parameter $k$ is increased [see Fig. 1(b)]. In contrast, however, a nonmonotonic behavior can take place with the lossy channel, as shown in Fig. 1(a). For low average photon numbers of each mode $N_{1}=N_{2}$, as in Figs. 1(b) and 1(c), a monotonic drop of the QCs occurs as the quantum efficiency $\eta$ decreases (we recall that the corresponding map reduces to the identity operator for $\eta=1)$. As the photon number becomes larger, though, the discord remarkably undergoes a rise so as to reach a maximum value and eventually drops to zero. This increase can be quite significant. For instance, as shown in Fig. 1(a), in the case $N_{1}=N_{2}=10$ the maximum attained by $\mathcal{D}$ is about 2.5 times the initial value (for $\eta=1$ ) and even $\simeq 10$ larger when $N_{1}=N_{2}=50$. For higher photon numbers (still keeping fixed the set value of $r$ ) the initial discord becomes extremely low, but a significant rise still takes place before the asymptotic decay. We illustrate the latter feature in the inset of Fig. 1(a) for the paradigmatic case $N_{1}=N_{2}=1000$, which corresponds to a fully separable state [28] with an initial discord $\left.\mathcal{D}\right|_{\eta=1} \simeq 10^{-6}$. Yet, under the lossy channel this undergoes a rise reaching a maximum $\simeq 0.5$ before the eventual slow decay. For all practical purposes, one can thus regard this behavior as the mere creation of (previously absent) quantum 

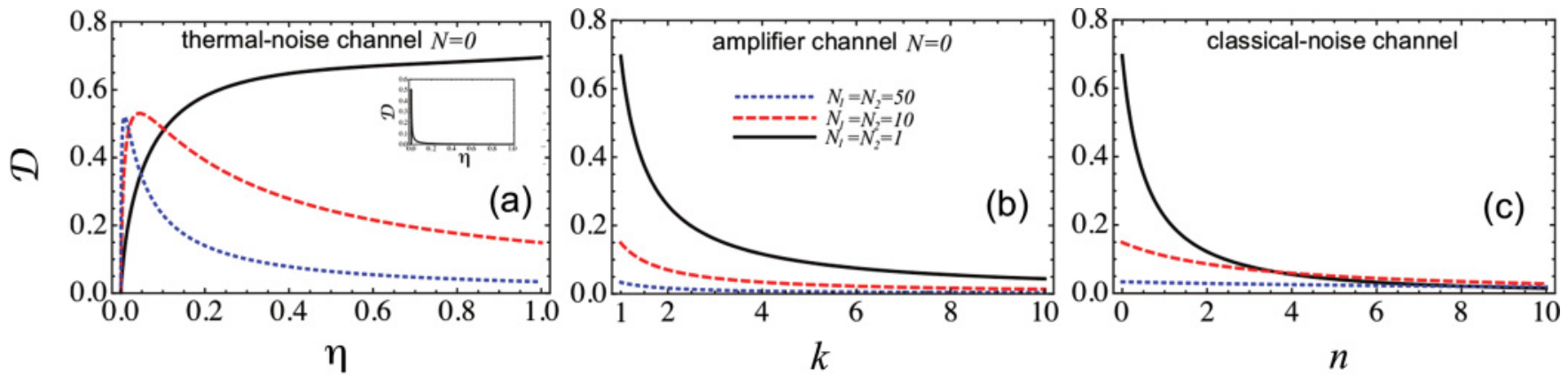

FIG. 1. (Color online) Behavior of Gaussian discord $\mathcal{D}$ under a thermal-noise (a), an amplifier (b), and a classical-noise (c) channel with the former two taken for $N=0$. Throughout, we have considered an initial state (7) for $r=1$ and $N_{1}=N_{2}=1$ (solid black lines), $N_{1}=$ $N_{2}=10$ (red dashed), and $N_{1}=N_{2}=100$ (blue dotted). The inset in (a) shows $\mathcal{D}$ against $\eta$ for $N_{1}=N_{2}=1000$ under the zero-temperature thermal-noise channel.

correlations, which is significantly reminiscent of an analogous effect occurring for qubits under local amplitude-damping channels [9-11].

It is natural to wonder how the discord rise is affected in the presence of a reservoir having nonzero temperature. To investigate this, in Fig. 2 we display $\mathcal{D}$ against $\eta$ in the case of the thermal-noise channel for growing values of the reservoir average photon number $N$. Clearly, the effect of temperature is to spoil the discord increase. At high enough temperatures, the initial rise is no longer exhibited and the behavior reduces to a mere monotonic decay similar to that in the amplifier and classical-noise channels [cf. Figs. 1(b) and 1(c)]. Note that there is a threshold value for $N$ separating the nonmonotonic regime (featuring a QC rise) from the monotonic one, the latter occurring above the threshold. For set $r$, this critical value grows linearly with $N_{1}$. This is shown in Fig. 3, where we plot the initial slope of the Gaussian discord $p$, i.e., $p=\partial \mathcal{D} / \partial \eta$ at $\eta=0$, vs $N$ and $N_{1}=N_{2}$. Recalling that $\eta$ decreases during the system's evolution under the thermal-noise channel, $p>0$ $(p<0)$ means that an initial decay (rise) of QCs occurs. Hence, the intersection of $p\left(N, N_{1}\right)$ with the $N-N_{1}$ plane provides the functional dependence of the aforementioned threshold value on $N_{1}$.

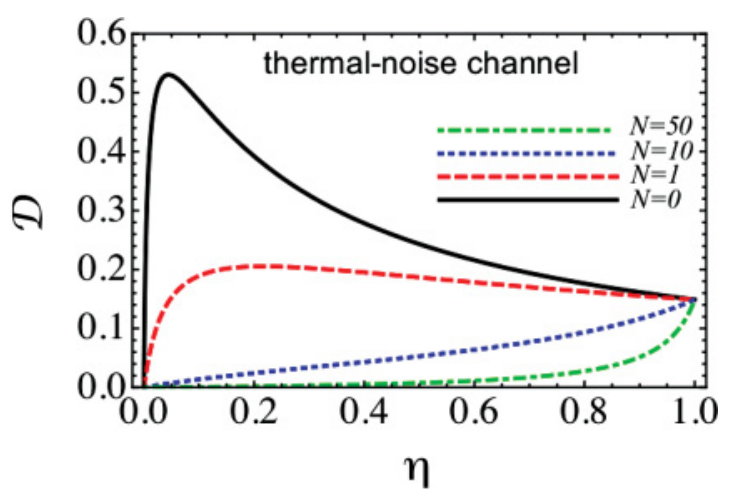

FIG. 2. (Color online) Gaussian discord $\mathcal{D}$ against $\eta$ under the thermal-noise channel for the average photon number of the reservoir $N=0$ (lossy channel, solid black line), $N=1$ (red dashed), $N=$ 10 (blue dotted), and $N=50$ (green dot-dashed). Throughout, the considered initial state is (7) with $r=1$ and $N_{1}=N_{2}=10$.

\section{INSIGHT INTO THE RISE OF QUANTUM CORRELATIONS}

Here, we give a picture that illustrates the emergence of some key features and behaviors presented in the previous section. We focus on the initial state specified by $r=1$ and $N_{1}=N_{2}=10$ exhibiting a rise of discord followed by a decay in the case of the thermal-noise channel (at low temperatures) and a monotonic drop with the other two channels (cf. Figs. 1 and 2). As in each of the three cases the environment does not affect the parameter $a^{\prime}$ [see Eqs. (16), (20), and (24)] and the equality $c_{1}^{\prime}=-c_{2}^{\prime}$ always holds, in the addressed regime the discord is in fact a function of $b^{\prime}$ and $c^{\prime}=\left|c_{1}^{\prime}\right|=\left|c_{2}^{\prime}\right|$ [cf. Eq. (5)]. As shown in the contour plot in Fig. 4(a), in its domain of definition $\mathcal{D}$ decreases with $b^{\prime}$ and increases with $c^{\prime}$. One can now obtain insight into the behavior of QCs by observing that the equations for $b^{\prime}$ and $c^{\prime}$ define a characteristic curve (associated with the specific channel) parametrized by $\eta \in[0,1], k>1$, and $n>0$ in the case of the thermal-noise, amplifier, and classical-noise channels, respectively. By expressing each of these parameters as a function of $c^{\prime}$ and replacing it into the equation for

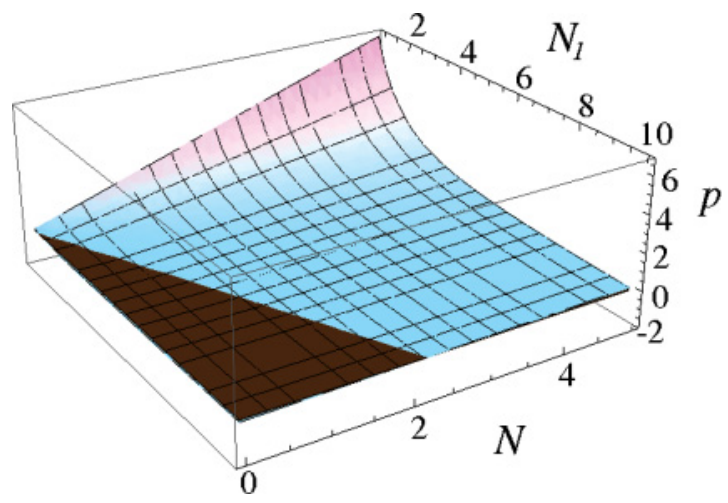

FIG. 3. (Color online) Initial slope of the Gaussian discord $p$, i.e., $\partial \mathcal{D} / \partial \eta$ at $\eta=0, \operatorname{vs} N$ and $N_{1}=N_{2} \eta$ for $r=1$ under the thermal-noise channel. The intersection line between the function profile (in blue) and the plane $p=0$ (in brown) specifies, for set $N_{1}$, the maximum value of $N$ yielding a rise in QCs. 

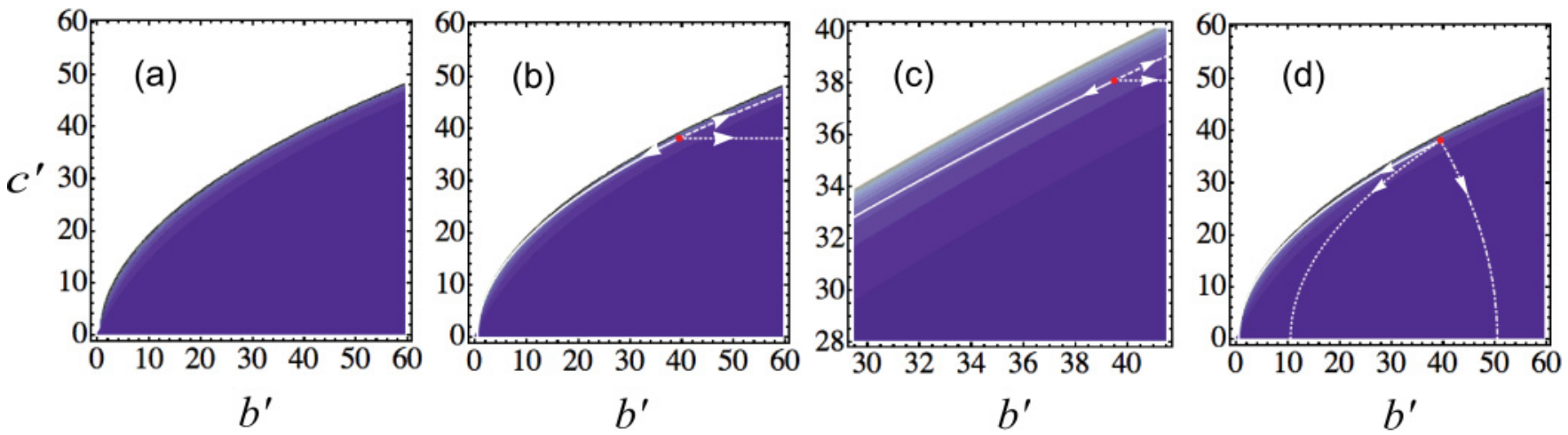

FIG. 4. (Color online) (a) Contour plot of Gaussian discord $\mathcal{D}$ against $b^{\prime}$ and $c^{\prime}$. (b) Oriented trajectories in the $b^{\prime} c^{\prime}$ plane arising with the lossy channel (solid line), the zero-temperature amplifier channel (dashed), and the classical-noise channel (dotted). The red point corresponds to the initial state. (c) Zoom of the previous figure highlighting the initial rise of discord occurring only under the lossy channel. (d) Effect of temperature in the case of the thermal-noise channel: oriented trajectories for $N=0$ (lossy channel, solid line), $N=1$ (dashed), $N=10$ (dotted), and $N=50$ (dot-dashed). Throughout, we have considered an initial state (7) for $r=1$ and $N_{1}=N_{2}=10$.

$b^{\prime}$, one obtains the trajectory of each channel in the $b^{\prime}-c^{\prime}$ plane as

$$
\begin{gathered}
b^{\prime}=\frac{b-(N+1 / 2)}{c^{2}} c^{\prime 2}+(N+1 / 2) \quad\left(c^{\prime} \in[0, c]\right), \\
b^{\prime}=\frac{b+(N+1 / 2)}{c^{2}} c^{\prime 2}-(N+1 / 2) \quad\left(c^{\prime} \in[c, \infty]\right), \\
c^{\prime}=c \quad\left(b^{\prime} \in[b, \infty]\right) .
\end{gathered}
$$

In Figs. 4(b) and 4(c), we report the above trajectories along with the same contour plot of discord as in Fig. 4(a). Evidently, due to the functional shape of $\mathcal{D}$ discussed above, a monotonic decrease of QCs necessarily takes place under the amplifier and classical-noise channels. It is also clear from the bottom left portion of Fig. 4(b) that for the lossy channel as $\eta$ is decreased $\mathcal{D}$ must eventually drop. However, the trajectory is such that over the first stage ( $\eta$ slightly below 1$)$ the QCs grow [this is best illustrated by the zoom presented in Fig. 4(c)].

The effect of temperature in the case of the thermalnoise channel (cf. Fig. 2) can be understood by scrutinizing Eq. (28) and Fig. 4(d). For $\eta \rightarrow 0 c^{\prime}$ tends to zero and thereby $b^{\prime} \rightarrow(N+1 / 2)$. Hence, the higher $N$ (i.e., the temperature) the larger the asymptotic value of $b^{\prime}$. Accordingly, the concavity $C=b-(N+1 / 2) / c^{2}$-which is positive for $N=0$ - progressively decreases so as to eventually become negative. As soon as it becomes small enough, the shape of $\mathcal{D}\left(b^{\prime}, c^{\prime}\right)$ [cf. Fig. 4(a)] prevents any initial rise from taking place, which results in a monotonic decay (see Fig. 2).

\section{CONCLUSIONS}

In this paper, we have investigated the behavior of Gaussian discord for a two-mode squeezed thermal state subject to various local Gaussian channels. Mainly motivated by the finding that a local amplitude-damping channel can create QCs in a pair of qubits, we have explored the dynamics under the thermal-noise, amplifier, and classical-noise channels. While the typical behavior is a monotonic decrease of QCs, as one would expect, we have found that in significant analogy with the qubit framework a thermal-noise channel can give rise to a nonmonotonic behavior comprising an initial rise of discord. For large enough photon numbers of each mode such that the initial QCs are in fact negligible, the above entails creation of previously absent $\mathrm{QCs}$ for all practical purposes. The reservoir temperature spoils this phenomenon in such a way that when it is high enough a mere monotonic decay occurs.

We have provided a picture that sheds light on various features, in particular the reason that the discord rise is exhibited only under the thermal-noise channel as well as the detrimental effect of temperature on it.

These findings significantly extend to the $\mathrm{CV}$ scenario one of the most counterintuitive effects entailed by the emerging extended paradigm of QCs: quantum correlations can be established in a composite system through interaction with a local and memoryless bath.

\section{ACKNOWLEDGMENTS}

We thank Matteo Paris and Mauro Paternostro for comments and acknowledge support from FIRB IDEAS through Project No. RBID08B3FM.
[1] M. A. Nielsen and I. L. Chuang, Quantum Computation and Quantum Information (Cambridge University Press, Cambridge, UK, 2000).

[2] R. Horodecki, P. Horodecki, M. Horodecki, and K. Horodecki, Rev. Mod. Phys. 81, 865 (2009).
[3] L. Henderson and V. Vedral, J. Phys. A 34, 6899 (2001); H. Ollivier and W. H. Zurek, Phys. Rev. Lett. 88, 017901 (2001).

[4] B. Groisman, D. Kenigsberg, and T. Mor, e-print arXiv: quant-ph/0703103. 
[5] J. Oppenheim, M. Horodecki, P. Horodecki, and R. Horodecki, Phys. Rev. Lett. 89, 180402 (2002); M. Horodecki, P. Horodecki, R. Horodecki, J. Oppenheim, A. Sen, U. Sen, and B. Synak-Radtke, Phys. Rev. A 71, 062307 (2005).

[6] A. Datta, A. Shaji, and C. M. Caves, Phys. Rev. Lett. 100, 050502 (2008); B. P. Lanyon, M. Barbieri, M. P. Almeida, and A. G. White, ibid. 101, 200501 (2008).

[7] T. Werlang, S. Souza, F. F. Fanchini, and C. J. Villas Boas, Phys. Rev. A 80, 024103 (2009); J. Maziero, L. C. Céleri, R. M. Serra, and V. Vedral, ibid. 80, 044102 (2009); L. Mazzola, J. Piilo, and S. Maniscalco, Phys. Rev. Lett. 104, 200401 (2010).

[8] A. Ferraro, L. Aolita, D. Cavalcanti, F. M. Cucchietti, and A. Acin, Phys. Rev. A 81, 052318 (2010).

[9] F. Ciccarello and V. Giovannetti, e-print arXiv:1105.5551.

[10] S. Campbell, T. J. G. Apollaro, C. Di Franco, L. Banchi, A. Cuccoli, R. Vaia, F. Plastina, and M. Paternostro, Phys. Rev. A 84, 052316 (2011).

[11] A. Streltsov, H. Kampermann, and D. Bruss, Phys. Rev. Lett. 107, 170502 (2011).

[12] X. Hu, Y. Gu, Q. Gong, and G. Guo, Phys. Rev. A 84, 022113 (2011).

[13] P. Giorda and M. G. A. Paris, Phys. Rev. Lett. 105, 020503 (2010).

[14] G. Adesso and A. Datta, Phys. Rev. Lett. 105, 030501 (2010).

[15] L. Mista Jr., R. Tatham, D. Girolami, N. Korolkova, and Gerardo Adesso, Phys. Rev. A 83, 042325 (2011).
[16] R. Vasile, P. Giorda, S. Olivares, M. G. A. Paris, and S. Maniscalco, Phys. Rev. A 82, 012313 (2010).

[17] A. Isar, Open Syst. Inf. Dyn. 18, 175 (2011).

[18] Here, we are not concerned with the open dynamics of two (or more) CV systems featuring a direct interaction between each other, which has recently been the focus of some studies.

[19] A. S. Holevo and R. F. Werner, Phys. Rev. A 63, 032312 (2001).

[20] J. Eisert and M. M. Wolf, Quantum Information with Continuous Variables of Atoms and Light (Imperial College Press, London, 2007), pp. 23-42.

[21] F. Caruso, J. Eisert, V. Giovannetti, and A. S. Holevo, New J. Phys. 10, 083030 (2008).

[22] G. Giedke and J. I. Cirac, Phys. Rev. A 66, 032316 (2002).

[23] D. F. Walls and G. J. Milburn, Quantum Optics (Springer, Berlin, 1994).

[24] A. Ferraro, S. Olivares, and M. G. A. Paris, Gaussian States in Continuous Variable Quantum Information (Bibliopolis, Napoli, 2005).

[25] V. Giovannetti, S. Guha, S. Lloyd, L. Maccone, and J. H. Shapiro, Phys. Rev. A 70, 032315 (2004).

[26] F. Caruso and V. Giovannetti, Phys. Rev. A 74, 062307 (2006).

[27] M. J. W. Hall and M. J. O. Rourke, Quantum Opt. 5, 161 (1993); M. J. W. Hall, Phys. Rev. A 50, 3295 (1994).

[28] Using the separability criterion in J. S. Prauzner-Bechcicki, J. Phys. A 37, L173 (2004), state (7) is separable whenever $N_{1} N_{2} /\left(1+N_{1}+N_{2}\right)>N_{r}$. 